\title{
Living donor liver transplantation for a patient with a history of total gastrectomy
}

\author{
Keita Shimata, Tomoaki Irie, Masashi Kadohisa, Seiichi Kawabata, Sho Ibuki, Yasuko Narita, \\ Hidekazu Yamamoto, Yasuhiko Sugawara*, Taizo Hibi \\ Department of Transplantation and Pediatric Surgery, Kumamoto University Graduate School of Medical Sciences, Kumamoto, \\ Japan.
}

\begin{abstract}
Summary Adhesions due to previous upper abdominal surgery may complicate later liver transplantation. Here we report successful living donor liver transplantation (LDLT) in a patient with a history of total gastrectomy. A 32-year-old Japanese woman developed end-stage liver failure due to alcoholic cirrhosis. She had undergone total gastrectomy, pancreato-splenectomy, and partial colectomy due to rupture of a pancreatic cyst. LDLT was performed using a right lobe graft from her sister. To minimize blood loss and injury to the jejunum, adhesions between the left lobe and nearby organs were dissected without blood flow in or out of the liver. The right liver graft was implanted uneventfully. She was extubated on postoperative day (POD) 1, but then developed septic shock due to aspiration pneumonia on POD 2. She was reintubated and antibiotics and antifungal agents were administered. Administration of tacrolimus was changed to an intravenous route on POD 3. Her condition improved and she was re-extubated on POD 9. On POD 14, tacrolimus was administered orally. She was discharged from our hospital on POD 30 without any other events and is doing well 6 months after LDLT. We believe that careful planning, such as mobilizing the left lobe with the blood flow blocked just before liver explantation, elevating the head of the bed during tube-feeding, and calculating the area under the curve after drug administration will enable liver transplantation for patients with a history of total gastrectomy.
\end{abstract}

Keywords: Total gastrectomy, adhesion, LDLT, aspiration pneumonia, tacrolimus

\section{Introduction}

The indications for liver transplantation (LT) in high-risk patients have been cautiously expanded due to improvements in LT techniques, including surgical techniques, perioperative management, immunosuppressive agents, and effective management of secondary complications. As a result, LT may be indicated in patients with significant comorbidities (1). In patients with a history of abdominal surgery, the operative procedures and postoperative management for

Released online in J-STAGE as advance publication April 15, 2019.

*Address correspondence to:

Dr. Yasuhiko Sugawara, Department of Transplantation and Pediatric Surgery, Kumamoto University Graduate School of Medical Sciences, Kumamoto, Japan.

E-mail: yasusuga-tky@umin.ac.jp
LT are often difficult due to intra-abdominal adhesions and distortion of bowel continuity, which may complicate liver explantation and biliary reconstruction (2-5). In addition, poor drug absorption in patients that have undergone gastric resection or a Roux-en-Y procedure may make immunosuppressive management difficult $(6,7)$. Although there are previous reports of LT after distal gastrectomy or sleeve gastrectomy as bariatric surgery $(5,8)$, there are no published reports describing LT in a patient with a history of total gastrectomy. Here we report successful living donor liver transplantation (LDLT) in a patient with a history of total gastrectomy, pancreato-splenectomy, and partial colectomy to treat a large pancreatic cyst rupture.

\section{Patients and Methods}

A 32-year-old Japanese woman developed end-stage 


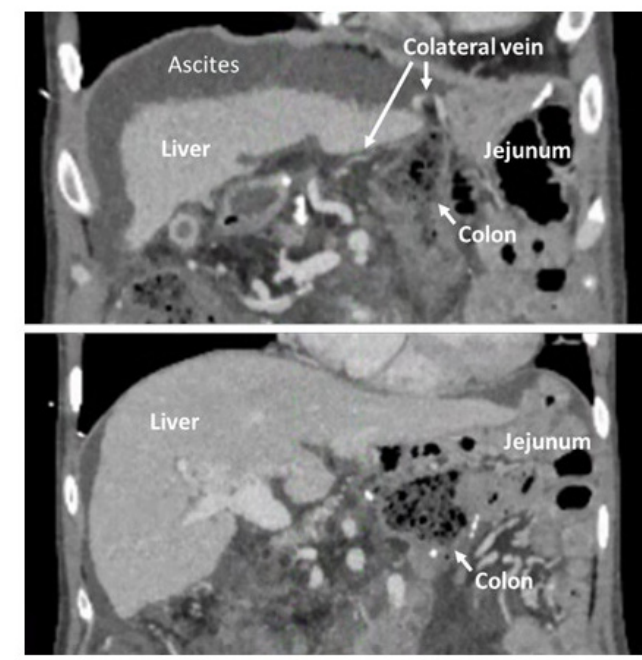

Figure 1. Preoperative angiographic computed tomography. These are coronal sections in the portal phase of preoperative angiographic computed tomography. These images showed severe adhesions between the left lobe and the Roux-Y jejunum or transverse colon, and collateral blood vessels around the left lateral segment.

liver failure due to alcoholic cirrhosis. She had a history of total gastrectomy, pancreato-splenectomy, and partial colectomy due to rupture of a pancreatic cyst. LDLT was indicated and a right lobe graft was obtained from her sister. Preoperative angiographic computed tomography showed severe adhesions between the left lobe and the Roux-en-Y jejunum, and collateral blood vessels at the portal vein and around the left lateral segment (Figure 1). Due to ABO bloodtype incompatibility between the donor and recipient, rituximab (400 mg/body, body weight: $50 \mathrm{~kg}$ ) was administered daily for 14 days before LDLT for B cell desensitization.

\section{Results and Discussion}

\subsection{Surgical procedure, and clinical course of the patient}

A reverse T-shaped incision was made with a longer midline incision to avoid the adhesions. At the upper left abdominal cavity, severe adhesions were observed between the liver and transverse colon. The hepatoduodenal ligament was dissected first, and then the hepatic arteries were divided and the portal vein dissected. Next, the right lobe of the liver was mobilized with the portal vein clamped. The short hepatic veins were separated from the right side by transecting the right hepatic vein because it was impossible to approach the site from left side due to adhesions between the jejunum and the left lobe of the liver. The middle and left hepatic vein were transected after clamping for subsequent anastomosis, followed by transection of the portal vein. Without blood flow

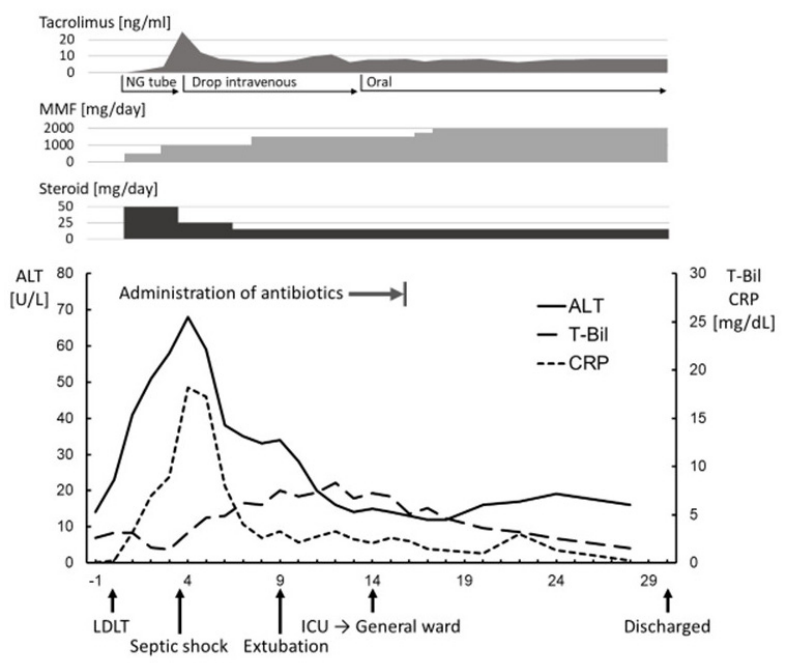

Figure 2. Postoperative clinical course. NG, nasogastric; MMF, mycophenolate mofetil; LDLT, living donor liver transplantation; ICU, intensive care unit

in or out of the liver, adhesions between the left lobe and the Roux-Y jejunum were dissected. In some areas with the tough adhesions, the liver parenchyma was resected using a vessel sealing system (Ligasure ${ }^{\mathrm{TM}}$ Small Jaw, Covidien, Japan) to prevent injuring the liver parenchyma covering the jejunum during surgery. Dissection of all adhesions around the liver was completed and the whole liver was explanted. A right liver graft (weight: $665 \mathrm{~g}$, graft to recipient body weight ratio: $1.33 \%$ ) with reconstruction of $\mathrm{V} 5+\mathrm{V} 8$ from the patient's sister was implanted in our regular fashion. No other events occurred throughout the LDLT procedure. The total blood loss was $15,780 \mathrm{~mL}$ and the operative time was $782 \mathrm{~min}$, including $204 \mathrm{~min}$ of functional anhepatic time after clumping the portal vein until reperfusion of the graft. After the operation, the patient was transferred to the intensive care unit on mechanical ventilation.

The postoperative course of the patient is shown in Figure 2. Immunosuppression was initiated with the administration of tacrolimus, steroids, and mycophenolate mofetil (MMF) using a nasogastric tube on postoperative day (POD) 1. This is our usual protocol for ABO blood type-incompatible LT. After extubating on POD 1, her respiratory condition worsened and she developed septic shock due to aspiration pneumonia. She was re-intubated on POD 2 and administered antibiotics (meropenem + vancomycin) and antifungal (micafungin) agents, as well as vasopressors and continuous hemodiafiltration with endotoxin absorption. To minimize the risk of aspiration and maintain the blood concentration of tacrolimus, administration of tacrolimus was changed to an intravenous route on POD 3. The target trough value was set to $8 \sim 10 \mathrm{ng} / \mathrm{mL}$. Her condition improved gradually, and she was re-extubated on POD 9. On POD 14, she was moved from the ICU to the general 
Table 1. Recommendations for liver transplantation in patients with a history of total gastrectomy

1) Mobilize the left lobe with the blood flow blocked just before liver explanation.

2) Place patients with a history of total gastrectomy in a head-up position while administering medicines or enteral nutrients.

3) Administer tacrolimus intravenously immediately after liver transplantation.

ward and tacrolimus was administered orally. She was discharged from the hospital on POD 30 and is doing well 6 months after LDLT.

She was administered $4 \mathrm{mg}$ of tacrolimus every $12 \mathrm{~h}$ and the blood concentration of tacrolimus was checked to determine the trough level at, $2 \mathrm{~h}, 3 \mathrm{~h}$, and 4 $\mathrm{h}$ after administration $(4.5 \mathrm{ng} / \mathrm{mL}, 25.1 \mathrm{ng} / \mathrm{mL}, 13.6 \mathrm{ng} /$ $\mathrm{mL}$, and $9.8 \mathrm{ng} / \mathrm{mL}$, respectively). The area under the curve (AUC) for the blood concentration of tacrolimus revealed a trough level of $200 \mathrm{ng}$ hour $/ \mathrm{mL}$; the trough level in patients with a total stomach is $5 \sim 7 \mathrm{ng} / \mathrm{mL}$. The trough level of MMF ranged from less than 0.4 to 1.83 $\mathrm{ng} / \mathrm{mL}$ when the patient was administered $1,000 \mathrm{mg}$ of MMF every $12 \mathrm{~h}$.

\subsection{Surgical technique in patients with severe adhesions}

LT for patients with a history of partial gastrectomy, especially sleeve gastrectomy, was recently reported $(2-5,8)$, but there are no reports of LT for patients with a history of total gastrectomy. Therefore, to our knowledge this is the first case of LDLT for a patient with a history of total gastrectomy. The successful outcome of the present case demonstrates the feasibility of LDLT as a treatment option for such patients.

With respect to the surgical techniques, mobilizing the left lobe with the blood flow blocked just before liver explantation effectively minimized blood loss during surgery. During LT, hyperfibrinolysis occurs at the anhepatic phase and immediately after reperfusion (9), when bleeding tends to increase. As such, minimizing bleeding before liver explantation is crucial. When performing LT in a patient with cirrhosis and a history of upper abdominal surgery, collateral vessel and adhesions around the liver may cause a large amount of intraoperative bleeding. A previous report described an intraoperative demise during $\mathrm{LT}$ after distal pancreatectomy (10). In the present case, dissection of adhesions around the left lateral lobe was performed just before explantation without blood flow in or out of the liver. Retrograde liver explantation, in which the transection of hepatic veins is performed before transection of all structures in the hepatoduodenal ligament, is effective in cases with shortening of the hepatoduodenal structure due to adhesions and sufficient collateral venous formation (8).

Intraoperative injuries to the intestine and colon when dissecting adhesions around the liver may cause severe complications in immunosuppressed patients after surgery. For this reason, when tough adhesions were dissected, the liver parenchyma was resected using a vessel sealing system and the jejunum was not injured due to the remnant liver parenchyma covering the jejunum.

Even if severe adhesions between the left lobe and the organ around the left lobe are predicted, elaborating on a surgical plan in advance can make LT possible for a patient with a history of upper abdominal surgery.

\subsection{Postoperative management of patients with total gastrectomy}

In the present case, aspiration pneumonia led to septic shock, likely due to absence of the gastric cardia and a strong immunosuppressive state were considered to be the reasons. Kaneda et al. reported that previous gastrectomy is a risk factor for postoperative pneumonia and recommended preoperative administration of ranitidine and antibiotics with a broader spectrum than that of cefazolin to prevent postoperative pneumonia (11). Elevating the head of the bed head during tubefeeding may also be effective for preventing aspiration (12). In light of our experience, patients with a history of total gastrectomy should be in a head-up position for a longer time and the injection rate should be decreased to avoid aspiration during administration of medicines or enteral nutrients.

Regarding immunosuppressive agents, drug absorption in patients without a stomach is unclear (6). The majority of oral tacrolimus absorption occurs in the proximal duodenum; however, it has been shown that the drug is absorbed from the intestine to the colon (7). In the present case, to keep the drug concentration high enough to prevent acute cellular rejection and to prevent aspiration, tacrolimus was administrated intravenously beginning on POD 3. After changing to oral administration of the drug, a higher dose of tacrolimus than usual was required in the present case to maintain an appropriate AUC of tacrolimus. MMF is primarily absorbed in the stomach (7), so the trough level of MMF was lower than expected in this case on the basis of other cases. Intravenous administration of tacrolimus and calculating the AUC by measuring the drug concentration at more than three points after administration is important for confirming the pharmacokinetics of immunosuppressive agents in patients with a history of total gastrectomy.

In conclusion, we believe that the three recommendations described in Table 1 enable LT in a patient with a history of total gastrectomy. 


\section{References}

1. Stauffer JA, Steers JL, Bonatti H, Dougherty MK, Aranda-Michel J, Dickson RC, Harnois DM, Nguyen JH. Liver transplantation and pancreatic resection: a singlecenter experience and a review of the literature. Liver Transpl. 2009; 15:1728-1737.

2. Taneja S, Gupta S, Wadhawan M, Goyal N. Single-lobe living donor liver transplant in a morbidly obese cirrhotic patient preceded by laparoscopic sleeve gastrectomy. Case Rep Transplant. 2013; 2013:279651.

3. Lin MY, Tavakol MM, Sarin A, Amirkiai SM, Rogers SJ, Carter JT, Posselt AM. Laparoscopic sleeve gastrectomy is safe and efficacious for pretransplant candidates. Surg Obes Relat Dis. 2013; 9:653-658.

4. Heimbach JK, Watt KD, Poterucha JJ, Ziller NF, Cecco SD, Charlton MR, Hay JE, Wiesner RH, Sanchez W, Rosen CB, Swain JM. Combined liver transplantation and gastric sleeve resection for patients with medically complicated obesity and end-stage liver disease. Am J Transplant. 2013; 13:363-368.

5. Safwan M, Collins KM, Abouljoud MS, Salgia R. Outcome of liver transplantation in patients with prior bariatric surgery. Liver Transpl. 2017; 23:1415-1421.

6. Chen L, Liberatore L, Chin T, Walker S, Fanous H, Nash MM, Rapi L, Huckle J, Zaltzman JS, Prasad GVR. The Impact of Total Gastrectomy on Pharmacokinetics in Kidney Transplant Immunosuppressive Drug Regimes: A Case Study. Transplantation. 2017; 101:2213-2217.

7. Rogers CC, Alloway RR, Alexander JW, Cardi M, Trofe
J, Vinks AA. Pharmacokinetics of mycophenolic acid, tacrolimus and sirolimus after gastric bypass surgery in end-stage renal disease and transplant patients: a pilot study. Clin Transplant. 2008; 22:281-291.

8. Eguchi S, Soyama A, Takatsuki M, Hidaka M, Adachi T, Kitasato A, Baimakhanov Z, Kuroki T. How to explant a diseased liver for living donor liver transplantation after previous gastrectomy with severe adhesion (with video). J Hepatobiliary Pancreat Sci. 2014; 21:E62-64.

9. Kim EH, Ko JS, Gwak MS, Lee SK, Kim GS. Incidence and clinical significance of hyperfibrinolysis during living donor liver transplantation. Blood Coagul Fibrinolysis. 2018; 29:322-326.

10. Van Vilsteren FG, Baskin-Bey ES, Nagorney DM, Sanderson SO, Kremers WK, Rosen CB, Gores GJ, Hobday TJ. Liver transplantation for gastroenteropancreatic neuroendocrine cancers: Defining selection criteria to improve survival. Liver Transpl. 2006; 12:448456.

11. Kaneda H, Nakano T, Taniguchi Y, Saito T, Konobu T, Saito Y. Impact of previous gastrectomy on postoperative pneumonia after pulmonary resection in lung cancer patients. Interact Cardiovasc Thorac Surg. 2012; 14:750753.

12. Scolapio JS. Decreasing aspiration risk with enteral feeding. Gastrointest Endosc Clin N Am. 2007; 17:711716 .

(Received February 19, 2019; Revised April 7, 2019; Accepted April 9, 2019) 